\title{
Embedded Trees and the Support of the ISE
}

\author{
Michael Drmota*
}

November 30, 2009

\begin{abstract}
Embedded trees are labelled rooted trees, where the root has zero label and where the labels of adjacent vertices differ (at most) by \pm 1 . Recently it was proved (see [6] and [9]) that the distribution of the maximum and minimum label are closely related to the support of the density of the integrated superbrownian excursion (ISE). The purpose of this paper is make this probabilistic limiting relation more explicit by using a generating function approach due to Bouttier, Di Francesco, and Guitter [5] that is based on properties of Jacobi's $\theta$ functions. In particular we derive an integral representation of the joint distribution function of the supremum and infimum of the support of the ISE in terms of the Weierstrass $\wp$-function. Furthermore we re-derive the limiting radius distribution in random quadrangulations (by Chassaing and Schaeffer [6]) with the help of exact counting generating functions.
\end{abstract}

\section{Introduction}

A planted plane tree is a rooted ordered tree, which means that all successors of a node have a left-to-right order. It is a classical result that the number $p_{n}$ of planted planted plane trees with $n$ edges equals the Catalan number

$$
p_{n}=\frac{1}{n+1}\left(\begin{array}{c}
2 n \\
n
\end{array}\right) \text {. }
$$

\footnotetext{
${ }^{1}$ Institute of Discrete Mathematics and Geometry, Vienna University of Technology, Wiedner Hauptstr. 8-10, A-1040 Wien, Austria,

michael.drmota@tuwien.ac.at, http://www.dmg.tuwien.ac.at/drmota/

The work of this author was supported by the Austrian Science Foundation FWF Grant No. S9604.
} 
An embedded tree (with increments \pm 1 or 0 and \pm 1 ) is a planted plane tree, where the vertices are labelled by integers such that the root has label 0 and labels of adjacent vertices differ by \pm 1 of 0 and \pm 1 (see Figure 1). By construction the numbers $q_{n}$ and $\bar{q}_{n}$ of different embedded trees (with increments \pm 1 or 0 and \pm 1 ) is given by

$$
q_{n}=2^{n} p_{n}=\frac{2^{n}}{n+1}\left(\begin{array}{c}
2 n \\
n
\end{array}\right) \quad \text { and } \quad \bar{q}_{n}=3^{n} p_{n}=\frac{2^{n}}{n+1}\left(\begin{array}{c}
2 n \\
n
\end{array}\right)
$$

In what follows we assume that every embedded tree (with $n$ edges) is equally likely. Of course, in this random setting every parameter on embedded trees becomes a random variable.
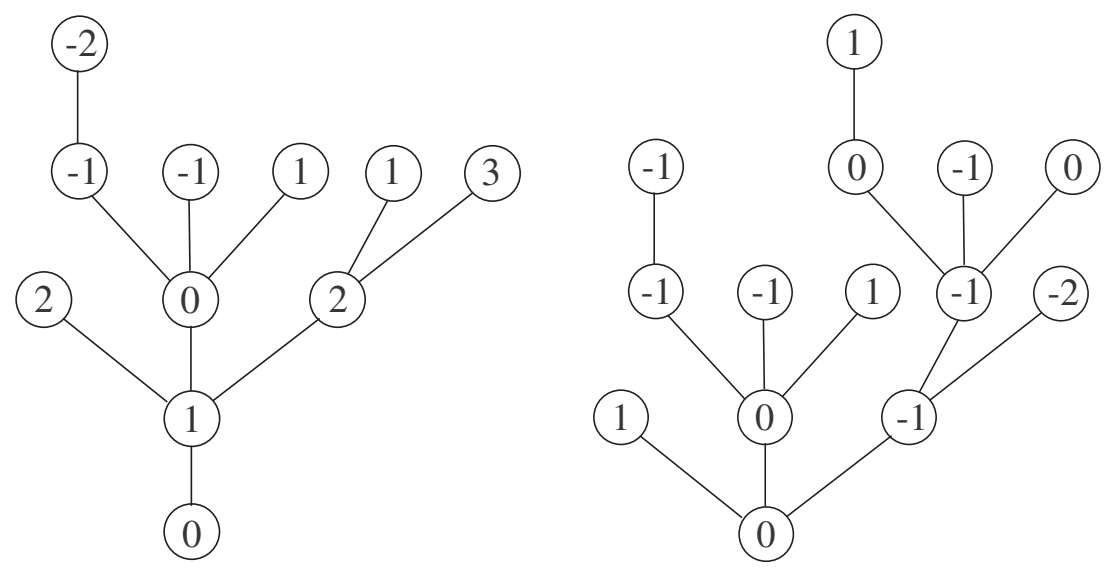

Figure 1: Embedded tree with increments \pm 1 and increments 0 and \pm 1

Let $X_{n}(j)$ denote the number of vertices with label $j$ in a (random) embedded tree of size $n$. The sequence $\left(X_{n}(j)\right)_{j \in \mathbb{Z}}$ is then the label profile, and let $X_{n}(t), t \in \mathbb{R}$, be the the linearly interpolated (random) function. Recently, Bousquet-Mélou and Janson [3] proved that

$$
\left(n^{-3 / 4} X_{n}\left(n^{1 / 4} t\right),-\infty<t<\infty\right) \stackrel{d}{\longrightarrow}\left(f_{\mathrm{ISE}}(t),-\infty<t<\infty\right)
$$

where $\stackrel{d}{\longrightarrow}$ denotes weak convergence in the space $\mathrm{C}_{0}(\mathbb{R})$ and the stochastic process $\left(f_{\mathrm{ISE}}(t),-\infty<t<\infty\right)$ is the densitiy of the integrated superbrownian excursion (ISE). Recall that the ISE is a random measure which can be 


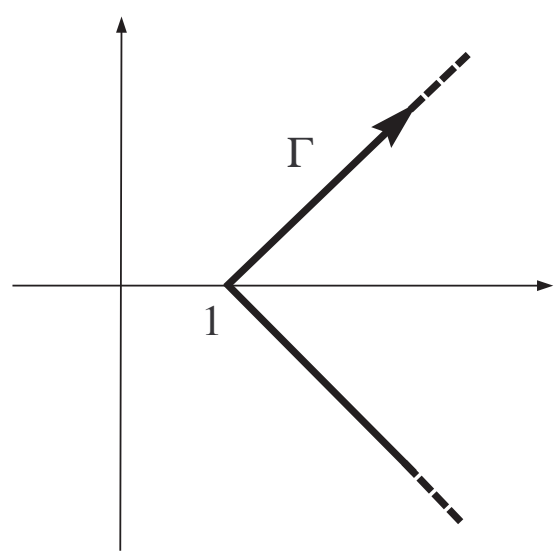

Figure 2: Contour of integration $\Gamma$

seen - despite of a scaling factor $\sqrt{2}$ - as the occupation measure of the head of the Brownian snake (see Chassaing and Schaeffer [6], Janson and Marckert [9], and Bousquet-Mélou and Janson [3]).

One interesting feature of the ISE is that the support of its density $\left[L_{\mathrm{ISE}}, R_{\mathrm{ISE}}\right]$ is (almost surely) a finite interval. By (1) it is clear that the largest label $M_{n}$ and the smallest label $m_{n}$ of a random embedded tree with $n$ edges is related to $R_{\mathrm{ISE}}$ and $L_{\mathrm{ISE}}$ :

$$
\frac{M_{n}}{n^{1 / 4}} \stackrel{d}{\longrightarrow} R_{\mathrm{ISE}} \quad \text { and } \quad \frac{m_{n}}{n^{1 / 4}} \stackrel{d}{\longrightarrow} L_{\mathrm{ISE}} .
$$

We also have

$$
\frac{M_{n}-m_{n}}{n^{1 / 4}} \stackrel{d}{\longrightarrow} R_{\mathrm{ISE}}-L_{\mathrm{ISE}} .
$$

Note that $R_{\mathrm{ISE}}$ and $-L_{\mathrm{ISE}}$ have the same distribution but they are not independent.

By using the relation between $M_{n}$ and $R_{\mathrm{ISE}}$ and asymptotics on generating functions Bousquet-Mélou [2] proved a remarkable integral representations of the tail distribution function $G(\lambda)=\mathbb{P}\left\{R_{\mathrm{ISE}}>\lambda\right\}$ :

$$
G(\lambda)=\frac{12}{i \sqrt{\pi}} \int_{\Gamma} \frac{v^{5} e^{v^{4}}}{\sinh ^{2}(\lambda v)} d v
$$

where

$$
\Gamma=\left\{1-t e^{-i \pi / 4}, t \in(-\infty, 0]\right\} \cup\left\{1+t e^{i \pi / 4}, t \in[0, \infty)\right\},
$$


see Figure 2. In [7] one can also find a relation for the Laplace transform of the function $x^{-\frac{3}{2}} \mathbb{P}\left\{R_{\mathrm{ISE}}>x^{\frac{1}{4}}\right\}$ which is given by

$$
\int_{0}^{\infty} x^{-\frac{3}{2}} \mathbb{P}\left\{R_{\mathrm{ISE}}>x^{\frac{1}{4}}\right\} e^{-s x} d x=\frac{6 \sqrt{\pi s}}{\left(\sinh \left((s / 2)^{1 / 4}\right)\right)^{2}}
$$

and representations for the moments

$$
\mathbb{E}\left(R_{\mathrm{ISE}}^{r}\right)=\frac{24 \sqrt{\pi} \Gamma(r+1) \zeta(r-1)}{2^{r} \Gamma((r-2) / 4)},
$$

for $\Re(r)>-4$, where the right hand side has to be analytically continued at the points $-3,-2,-1,2$.

The purpose of this paper is twofold. First we extend the result (3) by Bousquet-Mélou. We will provide integral representation for the joint distribution of $L_{\mathrm{ISE}}$ and $R_{\mathrm{ISE}}$ and also on the length $R_{\mathrm{ISE}}-L_{\mathrm{ISE}}$ of the support of the ISE. In the proof we use explicit representation of the corresponding generating function of embedded trees in terms of $\theta$-functions (see [5]) and asymptotics with Eisenstein series the Weierstrass $\wp$-function. The second purpose is to re-derive the limiting radius distribution of random quadrangulations by Chassaing and Schaeffer [6] with the help of exact counting generating functions. Here we make use of the well-known Schaeffer [11] bijection between quadrangulations and embedded trees with increments 0 and \pm 1 , where all labels are non-negative. A major property of this bijection is that the distances from the root vertex translate into the labels of the tree. Thus, the distance distribution persists, in particular the maximum distance from the root vertex (= radius of the quandrangulation) translates into the maximum label of the corresponding embedded tree.

The structure of the paper is as follows. In Section 2 we give precise statements of our results. The proof is then divided into two major part. First we discuss combinatorics on embedded trees (Section 3). In Section 4 we deal with the support of the ISE and in the final Section 5 with the length of the support of the ISE.

\section{Results}

As above let $M_{n}$ and $m_{n}$ denote the maximum and minimum labels in embedded trees of size $n$, respectively. In order to formulate our main result we 
need the notion of the Weierstrass $\wp$-function

$$
\wp(z ; \tau)=\frac{1}{z^{2}}+\sum_{\left(m_{1}, m_{2}\right) \in \mathbb{Z}^{2} \backslash\{(0,0)\}}\left(\frac{1}{\left(z-m_{1} \tau-m_{2}\right)^{2}}-\frac{1}{\left(m_{1} \tau+m_{2}\right)^{2}}\right),
$$

where $\tau$ and $z$ are complex variables with $\Im(\tau)>0$ and $z \notin \mathbb{Z}+\tau \mathbb{Z}$. The $\wp$-function - considered as a function in $z$ - is an elliptic function that has periods 1 and $\tau$. It is analytic in $\tau$ and meromorphic in $z$ with double poles on the lattice points $\mathbb{Z}+\tau \mathbb{Z}$; for details we refer to [10]. We will also need the notion of Eisenstein series

$$
G_{2 k}(\tau)=\sum_{\left(m_{1}, m_{2}\right) \in \mathbb{Z}^{2} \backslash\{(0,0)\}} \frac{1}{\left(m_{1}+m_{2} \tau\right)^{2 k}} .
$$

Theorem 2.1 The distribution function

$$
F\left(\lambda_{1}, \lambda_{2}\right)=\mathbb{P}\left\{R_{\mathrm{ISE}} \leq \lambda_{1},-L_{\mathrm{ISE}} \leq \lambda_{2}\right\}
$$

of the limit

$$
\left(\frac{M_{n}}{n^{1 / 4}}, \frac{-m_{n}}{n^{1 / 4}}\right) \stackrel{d}{\longrightarrow}\left(R_{\mathrm{ISE}},-L_{\mathrm{ISE}}\right)
$$

is given by

$$
F\left(\lambda_{1}, \lambda_{2}\right)=\frac{12}{i \pi^{5 / 2}} \int_{\Gamma} \wp\left(\frac{i \lambda_{1}}{\pi} v ; \frac{i\left(\lambda_{1}+\lambda_{2}\right)}{\pi} v\right) v^{5} e^{v^{4}} d v .
$$

This result can be used to obtain also a closed form expression for the distribution function of the length of the support $R_{\mathrm{ISE}}-L_{\mathrm{ISE}}$ by using the limit relation (2). However, we use a direct approach, where we re-derive a result of Chassaing and Schaeffer [6] on the radius of random quadrangulations.

Theorem 2.2 Let $r_{n}$ denote the maximum distance from the root vertex in random quadrangulations with $n$ faces. Then

$$
\gamma n^{-1 / 4} r_{n} \stackrel{d}{\longrightarrow} R_{\mathrm{ISE}}-L_{\mathrm{ISE}}
$$

where $\gamma=(3 / 2)^{-1 / 2}$. The distribution function of the limit is given by

$$
H(\lambda)=\mathbb{P}\left\{R_{\mathrm{ISE}}-L_{\mathrm{ISE}} \leq \lambda\right\}=\frac{1260}{i \pi^{13 / 2}} \int_{\Gamma} G_{6}\left(\frac{i \lambda}{\pi} v\right) v^{9} e^{v^{4}} d v .
$$


It seems that there is almost no literature on the explicit results on the support $\left[L_{\mathrm{ISE}}, R_{\mathrm{ISE}}\right]$ of the ISE. Besides the aboved mentioned results on $R_{\mathrm{ISE}}$ the expected values

$\mathbb{E}\left(-R_{\mathrm{ISE}} L_{\mathrm{ISE}}\right)=-3 \sqrt{2 \pi}+2 \sqrt{2 \pi} \int_{1}^{\infty} \int_{1}^{\infty} \frac{(u+1)}{\sqrt{t^{3}-1} \sqrt{u^{3}-1}\left(u+\sqrt{u^{2}+u+1}\right)} d u d t$

and

$$
\mathbb{E}\left(\min \left\{R_{\mathrm{ISE}},-L_{\mathrm{ISE}}\right\}\right)=6 \sqrt{2 \pi}\left(1-\frac{1}{8}\left(\int_{1}^{\infty} \frac{d u}{\sqrt{u^{3}-1}}\right)^{2}\right)
$$

have been computed by Delmas [7].

\section{Combinatorics}

\subsection{Planted Plane Trees}

Let $P(t)$ denote the generating function of planted plane trees, where the exponent of $t$ counts the number of edges. Then by using the combinatorial decomposition - namely that all subtrees of the root are again planted plane trees, see Figure 3 - we obtain the relation

$$
P(t)=1+t P(t)+t^{2} P(t)^{2}+t^{3} P(t)^{3}+\cdots=\frac{1}{1-t P(t)}
$$

and consequently

$$
P(t)=\frac{1-\sqrt{1-4 t}}{2 t}=\sum_{n \geq 0} \frac{1}{n+1}\left(\begin{array}{c}
2 n \\
n
\end{array}\right) t^{n} .
$$

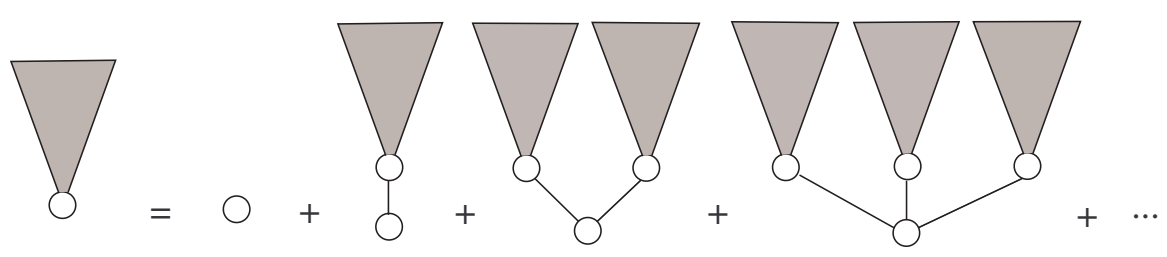

Figure 3: Recursion for planted plane trees 


\subsection{Embedded Trees with increments \pm 1}

It is also very easy to count embedded trees without any restriction with the help of generating functions. Let $R(t)$ denote the generating function of embedded trees, where the exponent of $t$ counts the number of edges. Furthermore let $R_{n}(t), n \in \mathbb{Z}$, be the generating function of embedded trees, where we assume that the root is labelled by $n$ (and labels of adjacent vertices differ by \pm 1 ). Then by using the same decomposition as above we have

$$
R_{n}(t)=\frac{1}{1-t\left(R_{n-1}(t)+R_{n+1}(t)\right)} .
$$

Since there are no restrictions on the embedded trees we have $R_{n}(t)=$ $R_{0}(t)=R(t)$ for all $n \in \mathbb{Z}$ leading to the relation

$$
R(t)=\frac{1}{1-2 t R(t)}
$$

and to the explicit representation

$$
R(t)=\frac{1-\sqrt{1-8 t}}{4 t}=\sum_{n \geq 0} \frac{2^{n}}{n+1}\left(\begin{array}{c}
2 n \\
n
\end{array}\right) t^{n} .
$$

The situation becomes more interesting if we just consider embedded trees, where all labels are non-negative. Let $R_{n}^{[0]}(t)$ be the generating functions of those embedded trees, where the root has label $n$. By definition $R_{n}^{[0]}(t)=0$ if $n<0$. However, we have the same recurrence relation as above:

$$
R_{n}^{[0]}(t)=\frac{1}{1-t\left(R_{n-1}^{[0]}(t)+R_{n+1}^{[0]}(t)\right)}, \quad(n \geq 0) .
$$

Interestingly, this system of equations has an explicit solution of the form

$$
R_{n}^{[0]}(t)=R(t) \frac{u_{n} u_{n+4}}{u_{n+1} u_{n+3}},
$$

where

$$
u_{n}=u_{n}(t)=Z(t)^{\frac{n+1}{2}}-Z(t)^{-\frac{n+1}{2}}
$$

and

$$
Z(t)=\frac{1-(1-8 t)^{1 / 4}}{1+(1-8 t)^{1 / 4}}
$$


is the solution of the equation

$$
Z+\frac{1}{Z}+2=\frac{1}{t R(t)}
$$

that is analytic at $t=0$. This miraculous relation was observed by Bouttier, Di Francesco, and Guitter [4]. In fact this explicit solution was used by Bousquet-Mélou [2] to obtain the integral representation for (3).

In another paper Bouttier, Di Francesco, and Guitter [5] considered the class of embedded trees, where all labels are bounded between 0 and $L$, where $L$ is a non-negative integer. Let $R_{n}^{[0, L]}(t)$ be the generating functions of those embedded trees, where the root has label $n$. By definition $R_{n}^{[0, L]}(t)=0$ if $n<0$ of $n>L$. As above we have the same recurrence relation as above:

$$
R_{n}^{[0, L]}(t)=\frac{1}{1-t\left(R_{n-1}^{[0, L]}(t)+R_{n+1}^{[0, L]}(t)\right)}, \quad(0 \leq n \leq L) .
$$

Interestingly there is an explicit solution of this system of equation in terms of the Jacobi theta function

$$
\theta_{1}(u ; q)=2 i \sin (\pi u) \prod_{j \geq 1}\left(1-2 q^{j} \cos (2 \pi u)+q^{2 j}\right)
$$

that we define here in an unnormalized form, see [5]. First let $q=q(t)$ be determined by the equation

$$
t=\frac{\theta_{1}\left(\frac{1}{L+6}, q\right)^{4} \theta_{1}\left(\frac{4}{L+6}, q\right)}{\theta_{1}\left(\frac{2}{L+6}, q\right)^{5}}
$$

Then we have (see [5])

$$
R_{n}^{[0, L]}(t)=\frac{\theta_{1}\left(\frac{2}{L+6}, q\right)^{3}}{\theta_{1}\left(\frac{1}{L+6}, q\right)^{2} \theta_{1}\left(\frac{4}{L+6}, q\right)} \frac{\theta_{1}\left(\frac{n+1}{L+6}, q\right) \theta_{1}\left(\frac{n+5}{L+6}, q\right)}{\theta_{1}\left(\frac{n+2}{L+6}, q\right) \theta_{1}\left(\frac{n+4}{L+6}, q\right)} .
$$

\subsection{Embedded Trees with increments 0 and \pm 1}

The equations for embedded trees with increments 0 and \pm 1 are very close to the previous ones.

Let $\bar{R}(t)$ denote the generating function of embedded trees, where the exponent of $t$ counts the number of edges. Furthermore let $\bar{R}_{n}(t), n \in \mathbb{Z}$, be 
the generating function of embedded trees, where we assume that the root is labelled by $n$ (and labels of adjacent vertices differ by 0 and \pm 1 ). Then we have

$$
\bar{R}_{n}(t)=\frac{1}{1-t\left(\bar{R}_{n-1}(t)+\bar{R}_{n}(t)+\bar{R}_{n+1}(t)\right)} .
$$

which leads to the relation

$$
\bar{R}(t)=\frac{1}{1-3 t \bar{R}(t)}
$$

and to the explicit representation

$$
\bar{R}(t)=\frac{1-\sqrt{1-12 t}}{6 t}=\sum_{n \geq 0} \frac{3^{n}}{n+1}\left(\begin{array}{c}
2 n \\
n
\end{array}\right) t^{n} .
$$

Similarly let $\bar{R}_{n}^{[0]}(t)$ be the generating functions of those embedded trees, where the root has label $n$. By definition $\bar{R}_{n}^{[0]}(t)=0$ if $n<0$. As above we get

$$
\bar{R}_{n}^{[0]}(t)=\frac{1}{1-t\left(\bar{R}_{n-1}^{[0]}(t)+\bar{R}_{n}^{[0]}(t)+\bar{R}_{n+1}^{[0]}(t)\right)}, \quad(n \geq 0) .
$$

and an explicit solution of the form

$$
\bar{R}_{n}^{[0]}(t)=\bar{R}(t) \frac{\bar{u}_{n} \bar{u}_{n+3}}{\bar{u}_{n+1} \bar{u}_{n+2}}
$$

where

$$
\bar{u}_{n}=\bar{u}_{n}(t)=\bar{Z}(t)^{\frac{n+1}{2}}-\bar{Z}(t)^{-\frac{n+1}{2}}
$$

and $\bar{Z}(t)$ is the solution of the equation

$$
\bar{Z}+\frac{1}{\bar{Z}}+4=\frac{1}{t \bar{R}(t)}
$$

that is analytic at $t=0$ (see [4]).

Next let $\bar{R}_{n}^{[0, L]}(t)$ be the generating functions of those embedded trees, where the root has label $n$ and all labels are bounded between 0 and $L$. Then we have (as above) $\bar{R}_{n}^{[0, L]}(t)=0$ if $n<0$ of $n>L$ and

$$
\bar{R}_{n}^{[0, L]}(t)=\frac{1}{1-t\left(\bar{R}_{n-1}^{[0, L]}(t)+\bar{R}_{n}^{[0, L]}(t)+\bar{R}_{n+1}^{[0, L]}(t)\right)}, \quad(0 \leq n \leq L) .
$$


The explicit solution of this system of equations is given by (see [5])

$$
\begin{aligned}
\bar{R}_{n}^{[0, L]}(t) & =4 \frac{\theta_{1}\left(\frac{1}{L+5}, q\right) \theta_{1}\left(\frac{2}{L+5}, q\right)}{\theta_{1}^{\prime}(0, q) \theta_{1}\left(\frac{3}{L+5}, q\right)}\left(\frac{\theta_{1}^{\prime}\left(\frac{1}{L+5}, q\right)}{\theta_{1}^{\prime}\left(\frac{1}{L+5}, q\right)}-\frac{\theta_{1}^{\prime}\left(\frac{2}{L+5}, q\right)}{2 \theta_{1}^{\prime}\left(\frac{2}{L+5}, q\right)}\right) \\
& \times \frac{\theta_{1}\left(\frac{n+1}{L+5}, q\right) \theta_{1}\left(\frac{n+4}{L+5}, q\right)}{\theta_{1}\left(\frac{n+2}{L+5}, q\right) \theta_{1}\left(\frac{n+3}{L+5}, q\right)},
\end{aligned}
$$

where $q=\bar{q}(t)$ be determined by the equation

$$
t=\frac{\theta_{1}^{\prime}(0, q)^{2} \theta_{1}\left(\frac{3}{L+5}, q\right)}{16 \theta_{1}\left(\frac{1}{L+5}, q\right) \theta_{1}\left(\frac{2}{L+6}, q\right)^{2}\left(\frac{\theta_{1}^{\prime}\left(\frac{1}{L+5}, q\right)}{\theta_{1}^{\prime}\left(\frac{1}{L+5}, q\right)}-\frac{\theta_{1}^{\prime}\left(\frac{2}{L+5}, q\right)}{2 \theta_{1}^{\prime}\left(\frac{2}{L+5}, q\right)}\right)^{2}}
$$

In particular we will be interested in the function

$$
\bar{R}_{0}^{[0, L]}(t)=4 \frac{\theta_{1}\left(\frac{1}{L+5}, q\right)^{2} \theta_{1}\left(\frac{4}{L+5}, q\right)}{\theta_{1}^{\prime}(0, q) \theta_{1}\left(\frac{3}{L+5}, q\right)^{2}}\left(\frac{\theta_{1}^{\prime}\left(\frac{1}{L+5}, q\right)}{\theta_{1}^{\prime}\left(\frac{1}{L+5}, q\right)}-\frac{\theta_{1}^{\prime}\left(\frac{2}{L+5}, q\right)}{2 \theta_{1}^{\prime}\left(\frac{2}{L+5}, q\right)}\right)
$$

which corresponds to embedded trees where all labels are non-negative and bounded by $L$. By the Schaeffer bijection this is also the generating function of rooted quadrangulations with $n$ faces, where all distances to the root are bounded by $L$.

\section{Asymptotic Analysis 1: Proof of Theorem 2.1}

In [5] the generating functions $R_{n}^{[0, L]}(t)$ have been analyzed by considering so-called scaling limits which can be interpreted in terms of potentials and characteristic lengths etc. For our purpose we have to be more precise, since we are interested in asymptotics of the coefficients. Nevertheless, we use more or less - the same of scaling as in [5].

By shifting labels from 0 to $j$ it follows that

$$
\mathbb{P}\left\{M_{n} \leq k, m_{n} \geq-j\right\}=\frac{\left[t^{n}\right] R_{j}^{[0, j+k]}(t)}{\frac{2^{n}}{n+1}\left(\begin{array}{c}
2 n \\
n
\end{array}\right)} .
$$

Thus, in order to prove Theorem 2.1 we need asymptotics on the coefficient $\left[t^{n}\right] R_{j}^{[0, j+k]}(t)$. Note that it is not necessary to prove asymptotics in the full 
range of parameters. In particular, we will set $j \sim \lambda_{1} n^{1 / 4}$ and $k \sim \lambda_{2} n^{1 / 4}$ for positive real numbers $\lambda_{1}, \lambda_{2}$.

We use Cauchy's formula

$$
\left[t^{n}\right] R_{j}^{[0, j+k]}(t)=\frac{1}{2 \pi i} \int_{\gamma} R_{j}^{[0, j+k]}(t) t^{-n-1} d t
$$

where $\gamma$ is a certain contour of winding number +1 around the origin, contained in the analyticity region of $R_{j}(t)^{[0, j+k]}$. In this case we will use a path of integration $\gamma$ of the form $\gamma=\gamma_{1} \cup \gamma_{2} \cup \gamma_{3} \cup \gamma_{4}$, where $x_{0}=\frac{1}{8}, c>0$,

$$
\begin{aligned}
& \gamma_{1}=\left\{x=x_{0}\left(1-c \frac{i+n^{1 / 4}-s}{n}\right): 0 \leq s \leq n^{1 / 4}\right\}, \\
& \gamma_{2}=\left\{x=x_{0}\left(1-c \frac{1}{n} e^{-i \varphi}\right):-\frac{\pi}{2} \leq \varphi \leq \frac{\pi}{2}\right\}, \\
& \gamma_{3}=\left\{x=x_{0}\left(1+c \frac{i+s}{n}:\right) 0 \leq s \leq n^{1 / 4}\right\},
\end{aligned}
$$

and $\gamma_{4}$ is a circular arc centred at the origin and making $\gamma$ a closed curve (see also Figure 4). Note that $\gamma_{1} \cup \gamma_{2} \cup \gamma_{3}$ constitutes a so-called Hankel contour that appears in Hankel's integral representation of $1 / \Gamma(s)$.
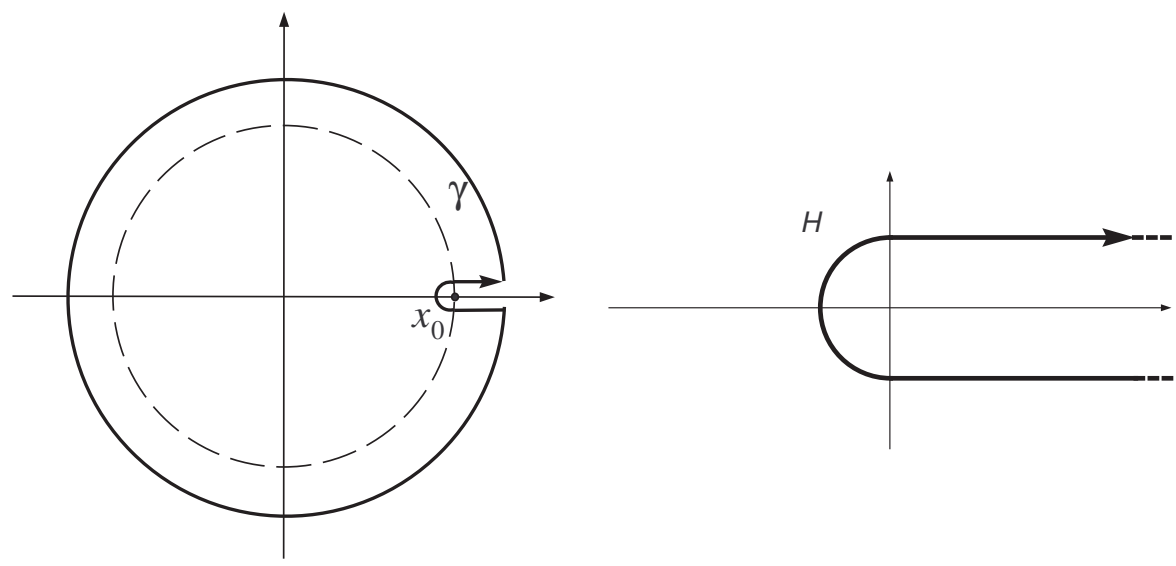

Figure 4: Contour of integration and Hankel contour

By the relation (9), $t$ and $q$ are related. We will first study this relation for $t \in \gamma_{1} \cup \gamma_{2} \cup \gamma_{3}$. For this purpose we first have to analyze $\theta_{1}(u, q)$. 
Lemma 4.1 We have for $u$ sufficiently close to 0 and for $|q|<1$

$$
\begin{aligned}
\theta_{1}(u, q) & =2 \pi i u \prod_{j \geq 1}\left(1-q^{j}\right)^{2 j} \\
& \times \exp \left(\left(-\frac{\pi^{2}}{6}+4 \pi^{2} \sum_{j \geq 1} \frac{q^{j}}{\left(1-q^{j}\right)^{2}}\right) u^{2}-\sum_{k \geq 2} \frac{u^{2 k}}{2 k} G_{2 k}(\tau)\right)
\end{aligned}
$$

where $q=e^{2 \pi i \tau}$ and $G_{2 k}(\tau)$ denote the Eisenstein series

$$
G_{2 k}(\tau)=\sum_{\left(m_{1}, m_{2}\right) \in \mathbb{Z}^{2} \backslash\{(0,0)\}} \frac{1}{\left(m_{1}+m_{2} \tau\right)^{2 k}} .
$$

Proof. Since $\wp(u, \tau)=-\log \left(\theta_{1}(u, q)\right)^{\prime \prime}+c$ (for $c=\theta_{1}^{\prime \prime \prime}(0, q) /\left(3 \theta_{1}^{\prime}(0, q)\right)$, see [1]) and the Laurent series expansion of $\wp(u, \tau)$ is given by

$$
\wp(u, \tau)=\frac{1}{u^{2}}+\sum_{k \geq 2}(2 k-1) u^{2 k-2} G_{2 k}(\tau)
$$

it follows that

$$
\log \theta_{1}(u, q)=c_{3}+c_{2} u+\frac{c}{2} u^{2}+\log u-\sum_{k \geq 2} \frac{u^{2 k}}{2 k} G_{2 k}(\tau)
$$

for some constants $c_{2}, c_{3}$. However, by using the Taylor series expansions for $\sin (\pi u)$ and $\cos (2 \pi u)$ we can represent $\theta_{1}(u, q)$ as

$\theta_{1}(u, q)=2 \pi i u \prod_{j \geq 1}\left(1-q^{j}\right)^{2 j} \cdot \exp \left(\left(-\frac{\pi^{2}}{6}+4 \pi^{2} \sum_{j \geq 1} \frac{q^{j}}{\left(1-q^{j}\right)^{2}}\right) u^{2}+O\left(u^{4}\right)\right)$.

Hence

$c=-\frac{\pi^{2}}{3}+8 \pi^{2} \sum_{j \geq 1} \frac{q^{j}}{\left(1-q^{j}\right)^{2}}, \quad c_{2}=0, \quad$ and $\quad c_{3}=\log \left(2 \pi i \prod_{j \geq 1}\left(1-q^{j}\right)^{2 j}\right)$,

which completes the proof of the lemma.

With the help of Lemma 4.1 we immediately obtain the following asymptotic representation. 
Lemma 4.2 Suppose that $q=e^{2 \pi i \tau}$ and L satisfy $|1-q| \geq c / L$ for some constant $c>0$. Then

$$
\begin{aligned}
& \frac{\theta_{1}\left(\frac{1}{L+6}, q\right)^{4} \theta_{1}\left(\frac{4}{L+6}, q\right)}{\theta_{1}\left(\frac{2}{L+6}, q\right)^{5}} \\
& \quad=\frac{1}{8}\left(1-\frac{45}{(L+6)^{4}} G_{4}(\tau)-\frac{630}{(L+6)^{6}} G_{6}(\tau)+O\left(\frac{1}{L^{8}|1-q|^{8}}\right)\right) .
\end{aligned}
$$

Next we discuss the behaviour of $G_{4}(\tau)$ if $\tau$ is close to 0 .

Lemma 4.3 We have uniformly for $\tau \rightarrow 0$ with $\varepsilon \leq \arg (\tau) \leq \pi-\varepsilon$ (for any $\varepsilon>0)$

$$
G_{4}(\tau)=\frac{\pi^{4}}{45} \tau^{-4}+O\left(\tau^{-3}\right)
$$

Proof. We use the alternate representation

$$
G_{4}(\tau)=\sum_{\left(m_{1}, m_{2}\right) \in \mathbb{Z}^{2} \backslash\{(0,0)\}} \frac{1}{\left(m_{1}+m_{2} \tau\right)^{4}}=\frac{\pi^{4}}{45}+\frac{16 \pi^{4}}{3} \sum_{n \geq 1} \sigma_{3}(n) e^{2 \pi i n \tau},
$$

where $\sigma_{\ell}(n)$ abbreviated $\sigma_{\ell}(n)=\sum_{d \mid n} d^{\ell}$. Hence, it suffices to consider the asymptotic properties of the sum

$$
S(x)=\sum_{n \geq 1} \sigma_{3}(n) e^{-n x}
$$

The Mellin transform of $S(x)$ (see [8]) is given by

$$
F(s)=\int_{0}^{\infty} S_{2}(x) x^{s-1} d x=\Gamma(s) \zeta(s) \zeta(s-3)
$$

for complex $s$ with $\Re(s)>4$. By taking the inverse Mellin transform (and shifting the line of integration to the left and taking into account the residue at $s=4$ ) one gets directly

$$
S(x)=\Gamma(4) \zeta(4) x^{-4}+O\left(x^{-3}\right)=\frac{\pi^{4}}{15} x^{-4}+O\left(x^{-3}\right)
$$

which is uniform for $x \rightarrow 0$ when $|\arg (x)| \leq \pi-\varepsilon$ for any $\varepsilon>0$ (see again [8]). Finally by using the relation $G_{4}(\tau) \sim(16 / 3) \pi^{4} S(-2 \pi i \tau)$ we obtain (19). 
We now assume that $L \sim j+k=\left(\lambda_{1}+\lambda_{2}\right) n^{1 / 4}$ for some positive constants $\lambda_{1}$ and $\lambda_{2}$. Furthermore it is convenient to introduce a new variable

$$
w=\frac{1}{2 \pi}(\log (1 / q))^{-1}=\frac{i}{\tau} .
$$

Now suppose that $t$ varies in $\gamma_{1} \cup \gamma_{2} \cup \gamma_{3}$ (with $x_{0}=\frac{1}{8}$ ). If we write $t=$ $\frac{1}{8}\left(1-\frac{w^{\prime}}{n}\right)$ then $w^{\prime}$ varies in $-H^{\prime}$, where $H^{\prime}$ is a Hankel contour cut at real part $n^{1 / 4}$. For simplicity we neglect this cut for a moment. With the help of the asymptotic relations of Lemmas 4.2 and 4.3 we have

$$
w^{\prime}=\frac{\pi^{4}}{\left(\lambda_{1}+\lambda_{2}\right)^{4}} w^{4}+O\left(w^{2}\right)
$$

Hence $w$ varies on a contour coming from $+e^{i \pi / 4} \infty$, cutting the real axis at some positive value and leaving to $+e^{-i \pi / 4} \infty$ (compare with Figure 5). Hence, without loss of generality we can assume that $w$ varies on $\hat{\Gamma}$, where $\Gamma$ is defined in (4) and ^denotes the time reversed contour.
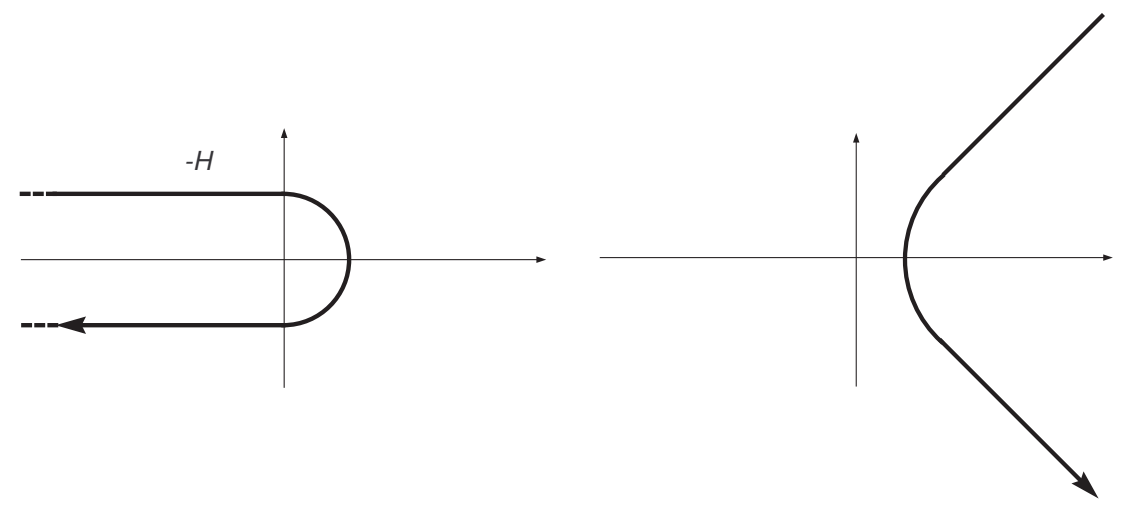

Figure 5: Negative Hankel contour and contour of integration of $w$

The next goal is to determine the asymptotic behaviour of $R_{j}^{[0, j+k]}(t)$ for $t \in \gamma_{1} \cup \gamma_{2} \cup \gamma_{3}$. For this purpose we will use the following property.

Lemma 4.4 Suppose that $q=e^{2 \pi i \tau}$ and L satisfy $|1-q| \geq c / L$ for some constant $c>0$. Then

$$
R_{j}^{[0, L]}(t)=2\left(1-\frac{3}{(L+6)^{2}} \wp\left(\frac{j+1}{L+6} ; \tau\right)+O\left(\frac{1}{L^{4}|1-q|^{4}}\right)\right)
$$

uniformly for $\varepsilon \leq j / L \leq 1-\varepsilon$ (for any $\varepsilon>0)$. 
Proof. By proceeding as in the proof of Lemma 4.2 we obtain

$$
\frac{\theta_{1}\left(\frac{2}{L+6}, q\right)^{3}}{\theta_{1}\left(\frac{1}{L+6}, q\right)^{2} \theta_{1}\left(\frac{4}{L+6}, q\right)}=2\left(1-\frac{1}{(L+6)^{2}} \frac{\theta_{1}^{\prime \prime \prime}(0, q)}{\theta_{1}^{\prime}(0, q)}+O\left(\frac{1}{L^{4}|1-q|^{4}}\right)\right) .
$$

Furthermore we have for $u=(j+1) /(L+6)$ (and uniformly for $\varepsilon \leq u \leq 1-\varepsilon$ )

$$
\frac{\theta_{1}\left(\frac{j+1}{L+6}, q\right) \theta_{1}\left(\frac{j+5}{L+6}, q\right)}{\theta_{1}\left(\frac{j+2}{L+6}, q\right) \theta_{1}\left(\frac{j+4}{L+6}, q\right)}=1+\frac{3}{(L+6)^{2}}\left(\frac{\theta_{1}^{\prime \prime}(u, q)}{\theta_{1}(u, q)}-\left(\frac{\theta_{1}^{\prime}(u, q)}{\theta_{1}(u, q)}\right)^{2}\right)+O\left(\frac{1}{L^{4}|1-q|}\right)
$$

Finally, by using the relation (see [1])

$$
\frac{\theta_{1}^{\prime \prime \prime}(0, q)}{3 \theta_{1}^{\prime}(0, q)}-\frac{\theta_{1}^{\prime \prime}(u, q)}{\theta_{1}(u, q)}+\left(\frac{\theta_{1}^{\prime}(u, q)}{\theta_{1}(u, q)}\right)^{2}=\wp(u ; \tau)
$$

we obtain the asymptotic relation (20).

We are now ready to prove Theorem 2.1. We set $j+3=\lambda_{1} n^{1 / 4}, k+3=$ $\lambda_{2} n^{1 / 4}$ and $L+6=(j+3)+(k+3)=\left(\lambda_{1}+\lambda_{2}\right) n^{1 / 4}$. As mentioned above we use Cauchy's formula. For technical reasons we apply it for $R_{j}^{[0, j+k]}(t)-2$ instead of $R_{j}^{[0, j+k]}(t)$. Of course, if $n>0$ we have

$$
\begin{aligned}
& {\left[t^{n}\right] R_{j}^{[0, j+k]}(t)=\left[t^{n}\right]\left(R_{j}^{[0, j+k]}(t)-2\right)=\frac{1}{2 \pi i} \int_{\gamma}\left(R_{j}^{[0, j+k]}(t)-2\right) t^{-n-1} d t } \\
= & \frac{1}{2 \pi i} \int_{\gamma_{1} \cup \gamma_{2} \cup \gamma_{3}}\left(R_{j}^{[0, j+k]}(t)-2\right) t^{-n-1} d t+\frac{1}{2 \pi i} \int_{\gamma_{4}}\left(R_{j}^{[0, j+k]}(t)-2\right) t^{-n-1} d t
\end{aligned}
$$

We will focus on the contribution coming from the contour $\gamma_{1} \cup \gamma_{2} \cup \gamma_{3}$. Namely if $t \in \gamma_{4}$ then $|t| \sim \frac{1}{8}\left(1+c n^{-3 / 4}\right)$ (for some $c>0$ ) whereas $R_{j}^{[0, j+k]}(t)$ stays bounded (note that Lemma 4.4 still applies). Hence

$$
\int_{\gamma_{4}}\left(R_{j}^{[0, j+k]}(t)-2\right) t^{-n-1} d t=O\left(8^{n} e^{-c n^{1 / 4}}\right)
$$

which is negligible compared to the normalization $\frac{2^{n}}{n+1}\left(\begin{array}{c}2 n \\ n\end{array}\right) \sim 8^{n} n^{-3 / 2} / \sqrt{\pi}$.

For $t \in \gamma_{1} \cup \gamma_{2} \cup \gamma_{3}$ we use the substitution

$$
t=\frac{1}{8}\left(1-\frac{\pi^{4} w^{4}}{L^{4}}\right)
$$


where $w=i / \tau$ now varies on a contour that we can deform (due to analyticity) to $\hat{\Gamma}$. Note, however, that we have to cut $\hat{\Gamma}$ to a finite contour $\hat{\Gamma}^{\prime}$, since $t \in \gamma_{1} \cup \gamma_{2} \cup \gamma_{3}$ implies that $w=O\left(n^{1 / 16}\right)$. In this range we use the approximations

$$
\begin{aligned}
R_{j}^{[0, j+k]}(t)-2 & =-\frac{6}{\left(\lambda_{1}+\lambda_{2}\right)^{2} \sqrt{n}} \wp\left(\frac{\lambda_{1}}{\lambda_{1}+\lambda_{2}} ; \frac{i}{w}\right)+O\left(\frac{w^{4}}{n}\right), \\
t^{-n-1} & =8^{n+1} \exp \left(\frac{\pi^{4}}{\left(\lambda_{1}+\lambda_{2}\right)^{4}} w^{4}+O\left(\frac{w^{6}}{\sqrt{n}}\right)\right),
\end{aligned}
$$

and the substitution

$$
d t=-\frac{1}{2} \frac{\pi^{4}}{\left(\lambda_{1}+\lambda_{2}\right)^{4} n} w^{3} d w
$$

that lead to the integral

$$
\begin{aligned}
& \frac{1}{2 \pi i} \int_{\gamma_{1} \cup \gamma_{2} \cup \gamma_{3}}\left(R_{j}^{[0, j+k]}(t)-2\right) t^{-n-1} d t=\frac{\pi^{4}}{2 \pi i} \frac{8^{n}}{n^{3 / 2}} \frac{12}{\left(\lambda_{1}+\lambda_{2}\right)^{6}} \\
& \quad \times \int_{\hat{\Gamma}^{\prime}}\left(\wp\left(\frac{\lambda_{1}}{\lambda_{1}+\lambda_{2}} ; \frac{i}{w}\right)+O\left(\frac{w^{4}+w^{6}}{\sqrt{n}}\right)\right) \exp \left(\frac{\pi^{4}}{\left(\lambda_{1}+\lambda_{2}\right)^{4}} w^{4}\right) w^{3} d w
\end{aligned}
$$

At this point we can neglect the error terms and extend the cutted path of integration $\hat{\Gamma}^{\prime}$ to the infinite path $\hat{\Gamma}$. Furthermore, we substitute $v=$ $\pi w /\left(\lambda_{1}+\lambda_{2}\right)$, use the relation $\wp(z ;-1 / \tau)=\tau^{2} \wp(z \tau ; \tau)$ and obtain (after reversing $\hat{\Gamma}$ to $\Gamma$ and deforming $\left(\pi /\left(\lambda_{1}+\lambda_{2}\right)\right) \Gamma$ to $\left.\Gamma\right)$

$$
\left[t^{n}\right] R_{j}^{[0, j+k]}(t) \sim \frac{12}{\pi^{3} i} \frac{8^{n}}{n^{3 / 2}} \int_{\Gamma} \wp\left(\frac{i \lambda_{1}}{\pi} v ; \frac{i\left(\lambda_{1}+\lambda_{2}\right)}{\pi} v\right) v^{5} e^{v^{4}} d v .
$$

Since $\frac{2^{n}}{n+1}\left(\begin{array}{c}2 n \\ n\end{array}\right) \sim 8^{n} n^{-3 / 2} / \sqrt{\pi}$ we finally derive the proposed result of Theorem 2.1 .

We note that the difference $M_{n}-m_{n}$ (and consequently the limit $R_{\mathrm{ISE}}-$ 
$L_{\mathrm{ISE}}$ ) can be handled with the help of the generating function

$$
\begin{aligned}
& \sum_{j+\ell \leq L}\left(R_{j}^{[0, \ell]}(t)-R_{j}^{[0, \ell-1]}(t)-R_{j-1}^{[0, \ell-1]}(t)+R_{j-1}^{[0, \ell-2]}(t)\right) \\
& =\sum_{j=0}^{L} \sum_{\ell=j \leq L}\left(R_{j}^{[0, \ell]}(t)-R_{j}^{[0, \ell-1]}(t)-R_{j-1}^{[0, \ell-1]}(t)+R_{j-1}^{[0, \ell-2]}(t)\right) \\
& =\sum_{j=0}^{L}\left(R_{j}^{[0, \ell]}(t)-R_{j-1}^{[0, \ell-1]}(t)\right),
\end{aligned}
$$

where we are interested in the $n$-th coefficient. However, this procedure is much more involved as that presented in the next section.

\section{Asymptotic Analysis 2: Proof of Theorem 2.2}

Let $r_{n}$ denote the maximum distance from the root vertex (= radius of the quandrangulation) which equals in distribution to the maximum label of embedded trees with increments 0 and \pm 1 , where all labels are non-negative.

Recall that all embedded trees with increments 0 and \pm 1 , where all labels are non-negative are counted by the generating function

$$
\begin{aligned}
\bar{R}_{0}(t) & =\bar{R}(t) \frac{(1-\bar{Z})\left(1-\bar{Z}^{4}\right)}{\left(1-\bar{Z}^{2}\right)\left(1-\bar{Z}^{3}\right)} \\
& =\bar{R}(t) \frac{1+\bar{Z}^{2}}{1+\bar{Z}+\bar{Z}^{2}} \\
& =\bar{R}(t)\left(1-t \bar{R}(t)^{2}\right)
\end{aligned}
$$

It is an easy exercise (by using Lagrange's inversion formula) to show that

$$
\left[t^{n}\right] \bar{R}_{0}(t)=\frac{2 \cdot 3^{n}}{(n+1)(n+2)}\left(\begin{array}{c}
2 n \\
n
\end{array}\right) \sim \frac{2 \cdot 12^{n}}{\sqrt{\pi}} n^{-5 / 2} .
$$

This is (of course) the classical formula for the number of quadrangulations with $n$ faces.

We also recall that the generating function $\bar{R}_{0}^{[0, L]}(t)$ (see (16), where $t$ and $q$ are related by (15)) corresponds to embedded trees with increments 0 and 
\pm 1 , where all labels are non-negative and bounded by $L$. Hence

$$
\mathbb{P}\left\{r_{n} \leq L\right\}=\frac{\left[t^{n}\right] \bar{R}_{0}^{[0, L]}(t)}{\frac{2 \cdot 3^{n}}{(n+1)(n+2)}\left(\begin{array}{c}
2 n \\
n
\end{array}\right)}
$$

In order to obtain an asmyptotic expansion for this probabilities we proceed as in the proof of Theorem 2.1. First we need an analogues to Lemma 4.2 and Lemma 4.4.

Lemma 5.1 Suppose that $q=e^{2 \pi i \tau}$ and $L$ satisfy $|1-q| \geq c / L$ for some constant $c>0$. Then

$$
\begin{aligned}
& \frac{\theta_{1}^{\prime}(0, q)^{2} \theta_{1}\left(\frac{3}{L+5}, q\right)}{16 \theta_{1}\left(\frac{1}{L+5}, q\right) \theta_{1}\left(\frac{2}{L+6}, q\right)^{2}\left(\frac{\theta_{1}^{\prime}\left(\frac{1}{L+5}, q\right)}{\theta_{1}^{\prime}\left(\frac{1}{L+5}, q\right)}-\frac{\theta_{1}^{\prime}\left(\frac{2}{L+5}, q\right)}{2 \theta_{1}^{\prime}\left(\frac{2}{L+5}, q\right)}\right)} \\
& =\frac{1}{12}\left(1-\frac{20}{(L+5)^{4}} G_{4}(\tau)-\frac{140}{(L+5)^{6}} G_{6}(\tau)+O\left(\frac{1}{L^{8}|1-q|^{8}}\right)\right) .
\end{aligned}
$$

Proof. In addition to the representation of $\theta_{1}(u, q)$ of Lemma 4.1 we use the expansion

$$
\frac{\theta_{1}^{\prime}(u, q)}{\theta_{1}(u, q)}=\frac{1}{u}-c u-\sum_{k \geq 2} u^{2 k-1} G_{2 k}(\tau)
$$

to obtain

$$
\begin{aligned}
\frac{\theta_{1}^{\prime}(u, q)}{\theta_{1}(u, q)}-\frac{1}{2} \frac{\theta_{1}^{\prime}(2 u, q)}{\theta_{1}(2 u, q)} & =\frac{3}{4}\left(\frac{1}{u}+\sum_{k \geq 2} \frac{4\left(4^{k-1}-1\right)}{3} u^{2 k} G_{2 k}(\tau)\right) \\
& =\frac{3}{4 u}\left(1+4 u^{4} G_{4}(\tau)+20 u^{6} G_{6}(\tau)+O\left(u^{8}\right)\right) .
\end{aligned}
$$

This leads to (22) immediately.

Lemma 5.2 Suppose that $q=e^{2 \pi i \tau}$ and L satisfy $|1-q| \geq c / L$ for some constant $c>0$. Then

$$
\bar{R}_{0}^{[0, L]}(t)=\frac{4}{3}\left(1-\frac{20}{(L+5)^{4}} G_{4}(\tau)-\frac{420}{(L+5)^{6}} G_{6}(\tau)+O\left(\frac{1}{L^{8}|1-q|^{8}}\right)\right)
$$


Proof. By using the relation (16) and by proceeding as in the proof of Lemma 5.1 we derive the result.

We are now ready to prove Theorem 2.2. We set $L+5=\lambda n^{1 / 4}$ and use Cauchy's formula. For technical reasons we apply it for $\bar{R}_{0}^{[0, L]}(t)-16 t$ instead of $\bar{R}_{0}^{[0, L]}(t)$. Of course, if $n>1$ we have

$$
\begin{aligned}
& {\left[t^{n}\right] \bar{R}_{0}^{[0, L]}(t)=\left[t^{n}\right]\left(\bar{R}_{0}^{[0, L]}(t)-16 t\right)=\frac{1}{2 \pi i} \int_{\gamma}\left(\bar{R}_{0}^{[0, L]}(t)-16 t\right) t^{-n-1} d t } \\
= & \frac{1}{2 \pi i} \int_{\gamma_{1} \cup \gamma_{2} \cup \gamma_{3}}\left(\bar{R}_{0}^{[0, L]}(t)-16 t\right) t^{-n-1} d t+\frac{1}{2 \pi i} \int_{\gamma_{4}}\left(\bar{R}_{0}^{[0, L]}(t)-16 t\right) t^{-n-1} d t
\end{aligned}
$$

We will focus on the contribution coming from the contour $\gamma_{1} \cup \gamma_{2} \cup \gamma_{3}$.

First, by combining Lemma 5.1 and Lemma 5.2 it follows that

$$
\bar{R}_{0}^{[0, L]}(t)-16 t=-\frac{1120}{3(L+5)^{6}} G_{6}(\tau)+O\left(\frac{1}{L^{8}|1-q|^{8}}\right)
$$

Hence, we using the corresponding substitutions (as in the proof of Theorem 2.1: $\left.\tau=i / w, t=\frac{1}{12}\left(1-\frac{4}{9} \frac{\pi^{4}}{L^{4}} w^{4}\right), \lambda=(L+5) n^{1 / 4}, v=(\pi / \lambda) w\right)$ we derive

$$
\begin{aligned}
\frac{1}{2 \pi i} \int_{\gamma_{1} \cup \gamma_{2} \cup \gamma_{3}} & \left(\bar{R}_{0}^{[0, L]}(t)-16 t\right) t^{-n-1} d t \\
& \sim \frac{\pi^{4}}{\pi i} \frac{12^{n}}{n^{5 / 2}} \frac{8960}{27 \lambda^{10}} \int_{\hat{\Gamma}} G_{6}\left(\frac{i}{w}\right) \exp \left(\frac{4}{9} \frac{\pi^{4}}{\lambda^{4}} w^{4}\right) w^{3} d w \\
& =\frac{1}{\pi i} \frac{12^{n}}{n^{5 / 2}} \frac{8960}{27 \lambda^{6}} \int_{\hat{\Gamma}} G_{6}\left(\frac{i \pi}{\lambda v}\right) \exp \left(\frac{4}{9} v^{4}\right) v^{3} d v \\
& =\frac{1}{\pi^{7} i} \frac{12^{n}}{n^{5 / 2}} \frac{8960}{27} \int_{\Gamma} G_{6}\left(\frac{i \lambda v}{\pi}\right) \exp \left(\frac{4}{9} v^{4}\right) v^{9} d v
\end{aligned}
$$

Hence, by normalizing with $2 \cdot 3^{n}\left(\begin{array}{c}2 n \\ n\end{array}\right) /((n+1)(n+2))$ we obtain the limting relation

$$
\mathbb{P}\left\{r_{n} \leq \lambda n^{1 / 4}\right\} \sim \frac{4480}{27} \frac{1}{\pi^{13 / 2} i} \int_{\Gamma} G_{6}\left(\frac{i \lambda v}{\pi}\right) \exp \left(\frac{4}{9} v^{4}\right) v^{9} d v
$$

A final scaling by $\sqrt{2 / 3}$ provides the result of Theorem 2.2 . 
Acknowledgement The author is grateful to Mireille Bousquet-Mélou for several helpful comments.

\section{References}

[1] H. Batman, Higher transcendental functions, vol. II, McGraw-Hill, New York, 1953.

[2] Bousquet-Mélou, M., Limit laws for embedded trees: applications to the integrated super-Brownian excursion, Random Structures Algorithms 29 (2006), 475-523.

[3] Bousquet-Mélou, M. and Janson, S., The density of the ISE and local limit laws for embedded trees, Ann. Appl. Probab. 16 (2006), 15971632.

[4] Bouttier, J. and Di Francesco, P. and Guitter, E., Geodesic distance in planar graphs, Nucl. Phys., B 663 (2003), 535-567.

[5] Bouttier, J. and Di Francesco, P. and Guitter, E., Random trees between two walls: exact partition function, J. Phys. A: Math. Gen. 36 (2003), 12349-12366.

[6] Chassaing, P. and Schaeffer, G., Random planar lattices and integrated superBrownian excursion, Probab. Theory Relat. Fields 128 (2004), $161-212$.

[7] J.-F. Delmas, Computation of moments for the length of the one dimensional ISE support, Electron. J. Probab. 8 (2003), Paper no. 17, 15 pp.

[8] Flajolet, P. and Gourdon, X. and Dumas, P., Mellin transforms and asymptotics: harmonic sums, Theoretical Computer Science 144 (1995), $3-58$.

[9] Janson, S. and Marckert, J.-F., Convergence of discrete snakes, J. Theor. Probab. 18 (2005), 615-645.

[10] M. Koecher and A. Krieg, Elliptische Funktionen und Modulformen, Springer, Berlin, 1998. 
[11] G. Schaeffer, Conjugaison darbres et cartes combinatoires aléatoires, PhD Thesis, Université Bordeaux I, 1998. 